\title{
A Mathematical Model of Multi-Hop HF Radio Propagation
}

\author{
Yaru Chen, Lu Han, Junrun Huang, Yufeng Gui* \\ College of Science, Wuhan University of Technology, Wuhan, China \\ Email: *guiyufeng@whut.edu.cn
}

How to cite this paper: Chen, Y.R., Han, L., Huang, J.R. and Gui, Y.F. (2018) A Mathematical Model of Multi-Hop HF Radio Propagation. Applied Mathematics, 9, 779-788.

https://doi.org/10.4236/am.2018.96054

Received: May 14, 2018

Accepted: June 26, 2018

Published: June 29, 2018

Copyright $\odot 2018$ by authors and Scientific Research Publishing Inc. This work is licensed under the Creative Commons Attribution International License (CC BY 4.0).

http://creativecommons.org/licenses/by/4.0/

c) (i) Open Access

\begin{abstract}
It is an eternal topic to study the minimum loss under the maximum transmission distance and the practicality of radio propagation in the navigation of ships. The transmission loss by dividing it to Median medium attenuation and Media attenuation correction factor is discussed. The Longley-Rice Model is introduced and improved; the correlation proportion of our model is work out. Then the reflection correction factor is calculated in the case of silent and turbulent ocean, which comes to the total loss and strength of the first reflection. The signal to noise ratio is explained and the relationship between various angle of incidence and correction factor are explored. According to fixed signal to noise ratio 14 , the highest bounce frequency is got.
\end{abstract}

\section{Keywords}

Longley-Rice, Radio Propagation, Ship, Mathematical Model, Random Waves

\section{Introduction}

Radio waves can travel long distances by multiple reflections off the ionosphere and off the earth with a high frequencies (HF, defined to be $3-30 \mathrm{mHz}$ ). HF radio waves from the ground whose frequencies are under maximum usable frequency (MUF) travel further and further with each successive hop by the reflections between the earth and the ionosphere again and again. And MUF has something to do with the season, time of day, and solar conditions. There is no reflection or refraction when the frequencies are over MUF. The characteristics of the reflecting surface determine the strength of the reflected wave and how far the signal will ultimately travel while maintaining useful signal integrity. The state of ocean also influences the attenuation of reflections. Ocean turbulence will affect the electromagnetic gradient of seawater, alter the local permittivity 
and permeability of the ocean, and chang the height and angle of the reflection surface.

We build a mathematical model based on Longley-Rice Model pinciple [1] for this signal reflection off the ocean [2] and then we simulate the three dimensional model of the waves [3].

For a 100-watt HF constant-carrier signal, below the MUF, from a point source on land, it determines the strength of the first reflection off a turbulent ocean and we can compare it with the strength of a first reflection off a calm ocean [4] to support that additional reflections ( 2 through $\mathrm{n}$ ) taking place off calm oceans [5] and working out the maximum number of hops the signal can take before its strength falls below a usable signal-to-noise ratio (SNR) threshold of $10 \mathrm{Db}$. Last we optimize the angle and find the maximum number of hops [4] [6].

Last but not least, the highlight of this paper is that the model draws on all the advantages of the previous semi-empirical model and more comprehensive consideration of various types of attenuation in transmission. Meanwhile [7] [8], according to the scope of the model we amend other factors, establish a highly generalized model of transmission loss and remain much to improve for more factors, which are continuous improvements of the growth mode [9] [10]. The model accurately describes the various types of losses in radio transmission acceptance, with great significance to the communication in the route [11].

\section{The Establishment of a Model}

\subsection{Radio Propagation Receiver}

The Longley-Rice radio wave transmission model is a radio wave transmission model proposed by Longley and Rice, which is a statistical model based on the radio wave propagation theory. And it combines many real-world measurement data, so the model is called semi-empirical prediction mode [7].

This model has a certain scope of application, so the decay needs to be corrected when the Medium attenuation, the introduction of correction factor. On the other hands, this model investigates radio waves as spherical emissions so we should sum each radio wave. As a result, our outcome should multiply by 4 .

\subsection{The Establish of Sealed Radio Propagation Receiver}

First of all, assuming that the frequency is $20 \mathrm{mHz}$, the attenuation of the radio wave in the first reflection is calculated by two parts of the medium median value and the medium attenuation correction factor [8].

In medium median, the information from references suggests that there is relevent. According to the different propagation range of radio waves, the transmission loss of Longley-Rice model can be divided into three cases: line-of-sight propagation loss, diffraction propagation loss and scattering propagation loss.

The transmission loss of the Longley-Rice model can be roughly divided into three cases: 1) line-of-sight propagation loss: $d_{\min } \leq d \leq d_{s}$. 
2) diffraction propagation loss: $d_{L s} \leq d \leq d_{x}$.

3) scattering propagation loss: $d_{x} \leq d$.

The propagation loss of radio waves in a calm sea surface is:

$$
\begin{gathered}
L_{b}=L_{r e f}+L_{\text {free }} \\
L_{\text {ref }}(d)=\left\{\begin{array}{lc}
\max \left(0, L_{b e}+k_{1} d+k_{2} \lg d\right) & d_{\min } \leq d \leq d_{x} \\
L_{b e d}+m_{d} d & d_{l s} \leq d \leq d_{x} \\
L_{b e s}+m_{s} d & d_{x} \leq d
\end{array}\right.
\end{gathered}
$$

$d$ is the propagation distance in $\mathrm{km}$;

$f$ is the radio wave frequency in $\mathrm{MHz}$;

$d_{L s}$ is the Smooth ground distance;

$d_{x}$, where the diffraction loss and scattering loss are equal;

$L_{b e}, L_{b e d}, L_{b e s}$ represents the value of propagation loss in line-of-sight, diffraction, and scattering under free space;

$k_{1}$ and $k_{2}$ are the propagation loss coefficients;

$m_{d}$ and $m_{s}$ are the diffraction and scattering loss coefficients, respectively.

Since this formula is a semi-empirical and the unknown coefficient is complicated, a large amount of data is collected according to the actual situation to be fitted.

We have:

$$
L=\left\{\begin{array}{cc}
0.8677 d+93.31 & d \leq 56 \\
0.6073 d+108.9 & 56 \leq d \leq 136 \\
0.1363 d+172.9 & d \geq 136
\end{array}\right.
$$

Then we come to multi-hop: Known elevation can be based on geometric knowledge to get the relationship between the hopping angle and angle of incidence as shown in Figure 1, we take the ionosphere height $h=100 \mathrm{~km}$ and the Earth's radius $R=6371 \mathrm{~km}$.

We have:

$$
d=2 R\left(\arccos \left(\frac{R \cos \partial}{h+R}\right)-\partial\right)
$$

\subsection{Correction of Attenuation in Medium}

1) Since radio waves propagate in the line of sight range, the radio wave propagation mode is mainly diffractive propagation, and the subject requires the study of reflection. Therefore, it is not necessary to discuss the diffraction correction of the sea surface within the viewing distance range.

2) The following picture suggest that when a point light emits signals in all directions, the energy of all the electric waves that can reach (3) only comes from the loss of electric wave propagation in the area (2), and the energy loss of the electric waves emitted in the range (1) is not considered. For that case the coefficients need to be corrected with correction ratio which is mean of angle, see Figure 2.

Since the corresponding propagation distance in scattering is infinite, all 


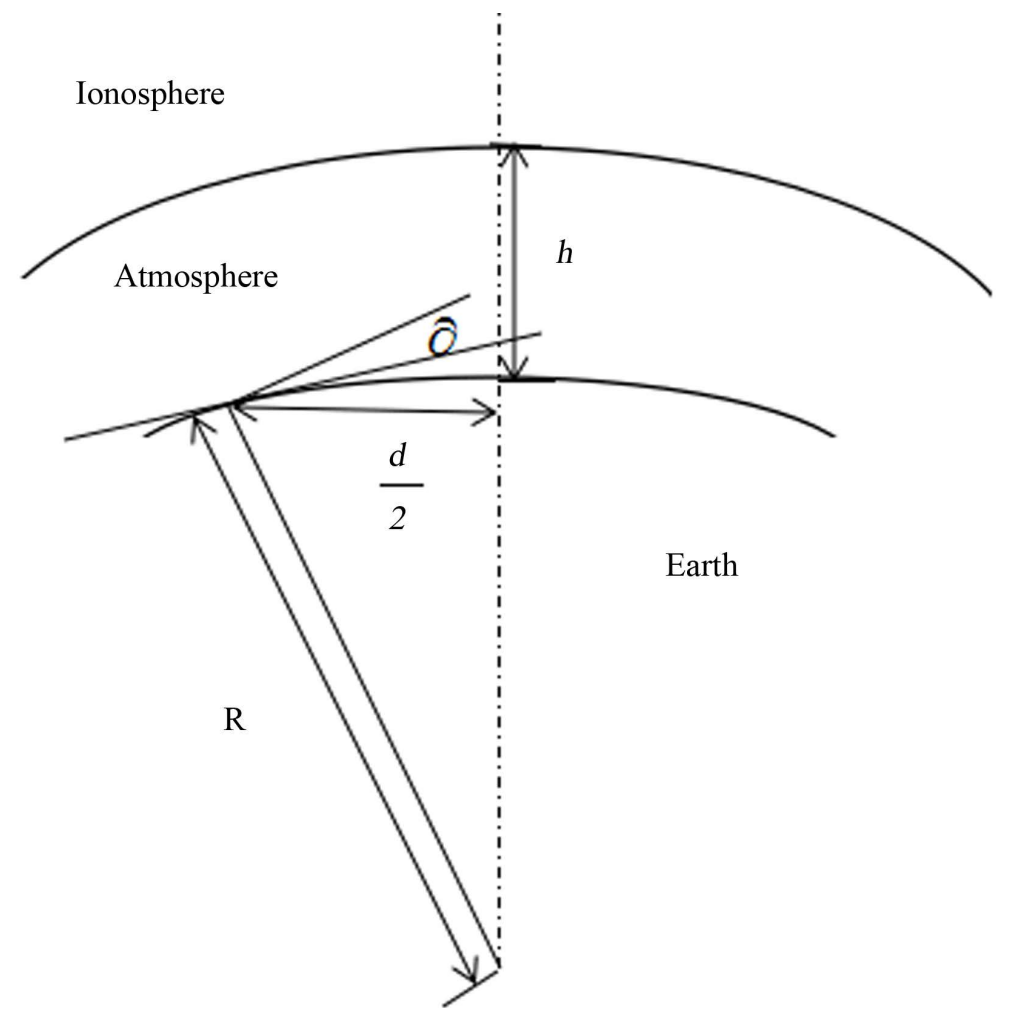

Figure 1. Earth and atmosphere.

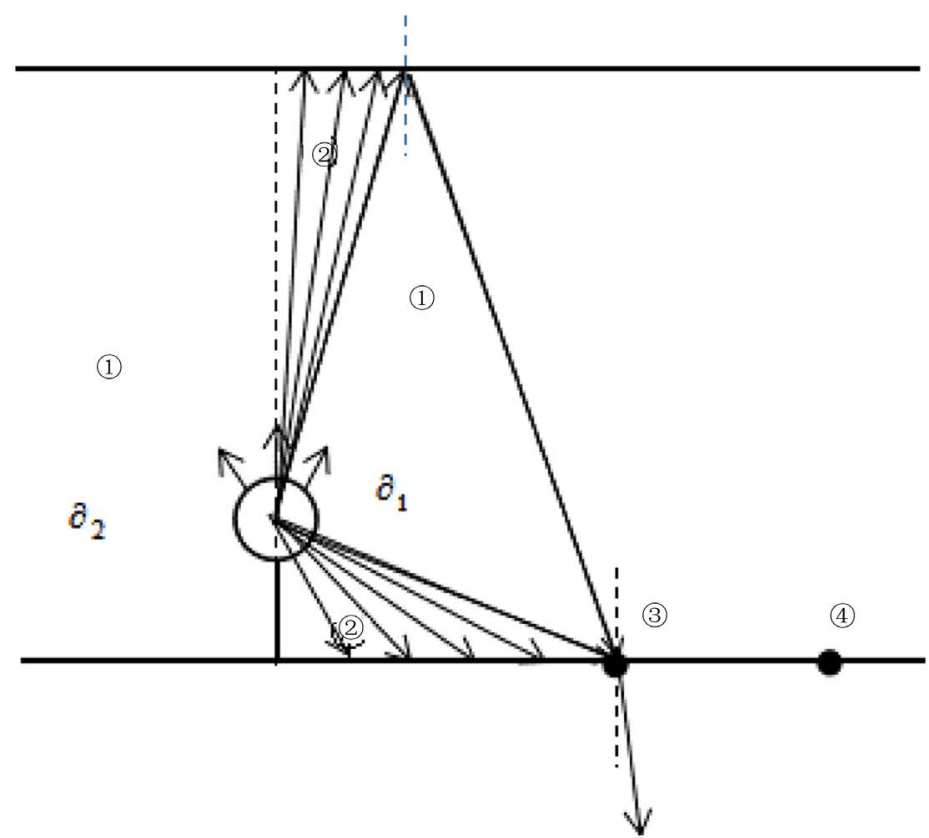

Figure 2. Relation of angles.

signal losses are taken into account, so there is no need to correct the scattering losses.

3) The calm sea surface reflection coefficient by the law of refraction refraction. Because of the law of refraction refraction: $n_{1} \theta_{i}=n_{2} \theta_{i}$. 
We calculate the reflection coefficient: with:

$$
\begin{gathered}
Q_{1}=L \times 4 \pi d^{2} \times R_{1} \\
R_{1}=\frac{1}{2}\left(r_{1}^{2}+r_{2}^{2}\right), r_{1}=\frac{\lambda_{2} \cos \theta_{i}-\lambda_{1} \cos \theta_{t}}{\lambda_{2} \cos \theta_{i}+\lambda_{1} \cos \theta_{t}} \\
r_{2}=\frac{\lambda_{1} \cos \theta_{i}-\lambda_{2} \cos \theta_{t}}{\lambda_{1} \cos \theta_{i}+\lambda_{2} \cos \theta_{t}}, \lambda_{i}=\sqrt{\frac{\mu_{i}}{\varepsilon_{i}}}(i=1,2)
\end{gathered}
$$

The relationship between the reflection coefficient and the incident angle is shown in Figure 3.

Based on the empirical formula in the longley-Rice model, we can deduce that the attenuation of the first reflection at calm sea surface is:

4) Rough turbulent ocean surface reflection coefficient correction

(1) The establishment of 3D turbulent ocean wave model based on ocean wave spectrum.

Because there is sea slope in turbulent sea compared to the calm sea surface, a three-dimensional turbulent ocean wave model needs to be established to study it.

(2) Create a wave model

The wave is described by a stationary stochastic process with ergodicity. The wave is viewed as a superposition of waves and swells in a simple cosine wave of infinitely different amplitudes, of varying frequency and of an incipient phase. That is, for the composition of wave propagation direction relative to the wind direction angle. $\theta$ is the angle which wave spread by in $x$. We use the wave spectrum function in reference:

$$
S(\rho, \theta)=\frac{8.1 \times 10^{-3} g^{2}}{w^{5}} \exp \left[-0.74\left(\frac{g}{u w}\right)^{4}\right] \frac{2}{\pi} \cos ^{2}(\theta)
$$

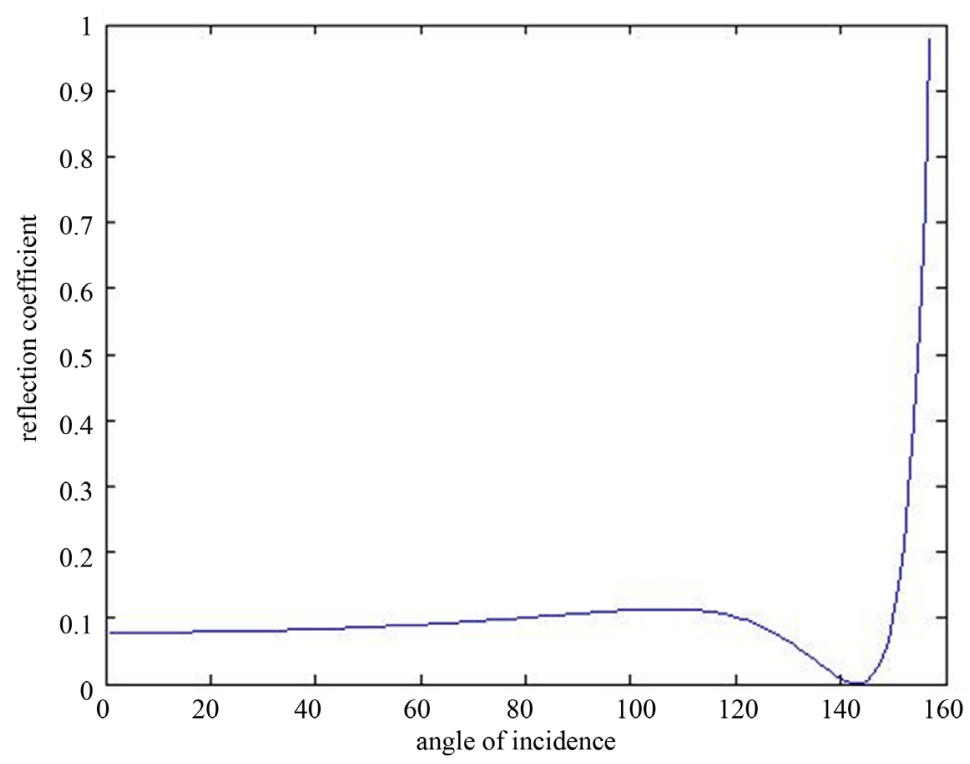

Figure 3. The relationship between angle and reflection confficient. 


$$
S(\rho, \theta)=\frac{8.1 \times 10^{-3} g^{2}}{w^{5}} \exp \left[-0.74\left(\frac{g}{u w}\right)^{4}\right] \frac{8}{3 \pi} \cos ^{2}(\theta)
$$

Create a random wave model shown in Figure 4.

From this model, we get the slope distribution function of each discrete sea surface randomly according to the wave spectrum (Figure 5).

(3) Set up the sea coordinate system

Set up the sea coordinate system as shown. Let the origin be located on the sea surface where the study object is located. The $\mathrm{X}$ and $\mathrm{Y}$ axes are located on the horizontal plane of the coordinate system. The positive direction of $\mathrm{Y}$ axis is the position of the detector and the positive direction of $\mathrm{Z}$ axis is upwar, see Figure 6.

We change the coordinates, maintaining the $\mathrm{Z}$ axis direction unchanged. The coordinate system is rotated clockwise:

$$
\left\{\begin{array}{l}
z_{u}=S_{x} \cos \gamma+S_{y} \sin \gamma \\
z_{v}=-S_{x} \cos \gamma+S_{y} \sin \gamma
\end{array}\right.
$$

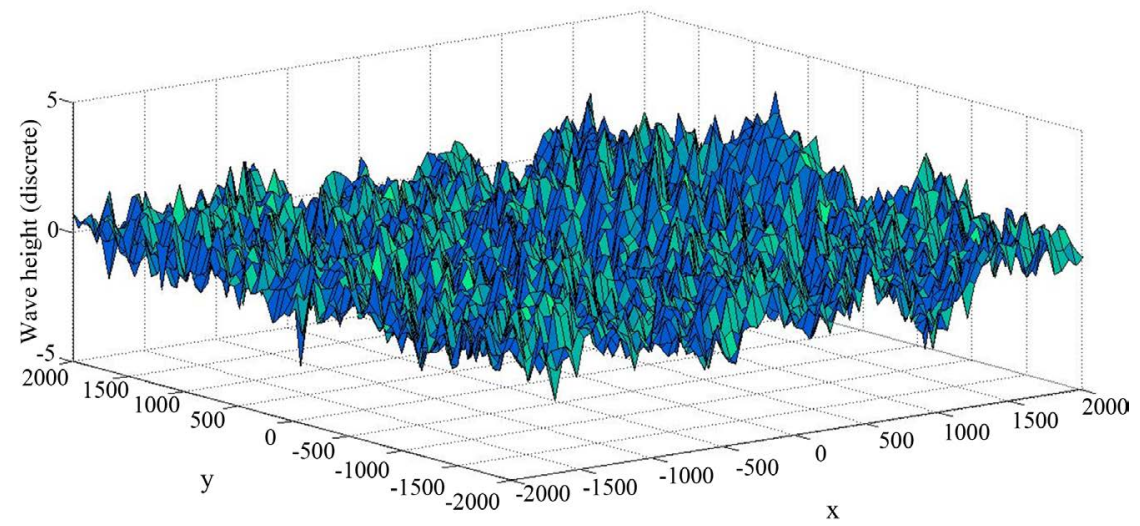

Figure 4. Wave mode.

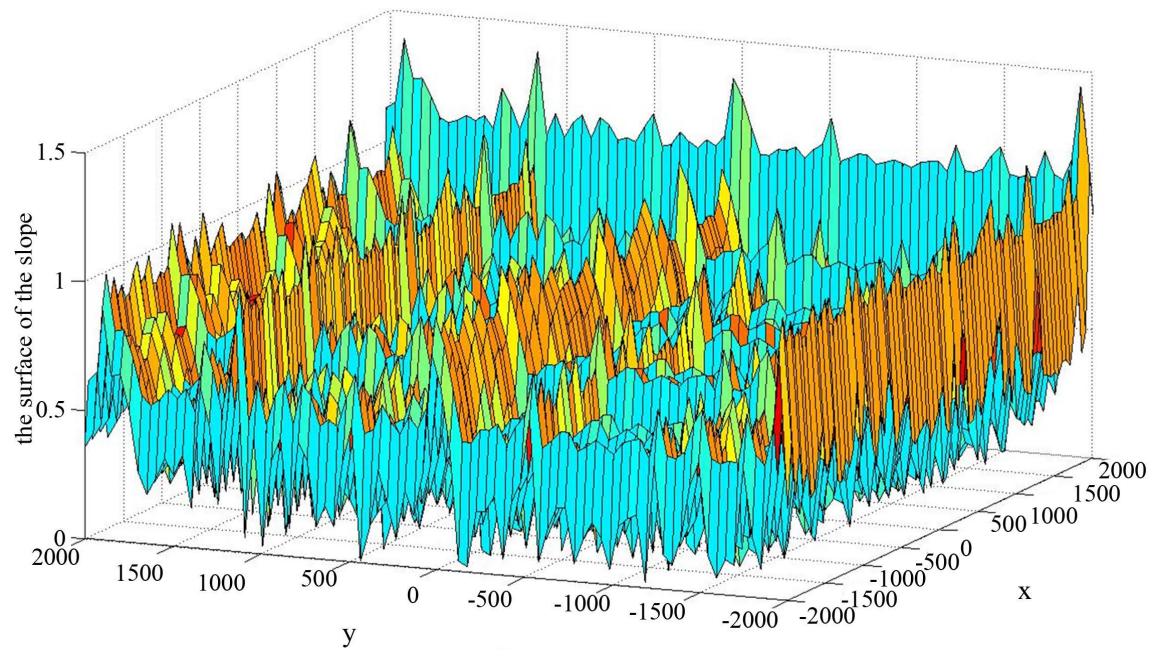

Figure 5. Spectrum. 
Roughness correction factor $\xi$ :

$$
\xi=\frac{\pi P\left(z_{x}, z_{y}\right)}{4 \cos \omega_{i} \cos ^{4} \beta}
$$

Rough correction factor and the relationship between the incidence angle shown in Figure 7.

Because: The reflection coefficient on the turbulent sea is the reflection coefficient on calm sea surface with rough correction factor.

The reflection coefficient on the turbulent sea is:

$$
R_{2}=\frac{\pi P\left(z_{x}, z_{y}\right) R_{1}}{4 \cos \omega_{i} \cos ^{4} \beta}
$$

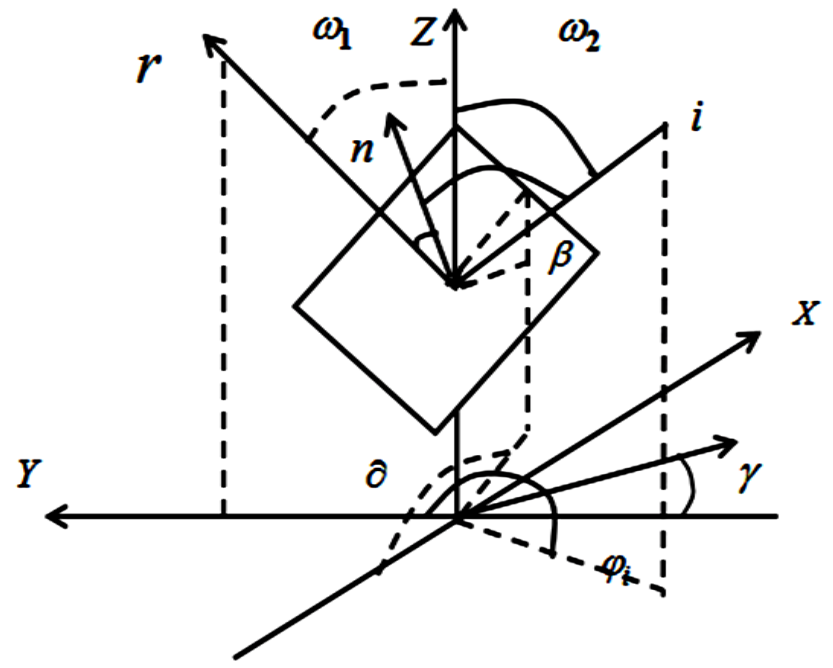

Figure 6. Relation of angle.

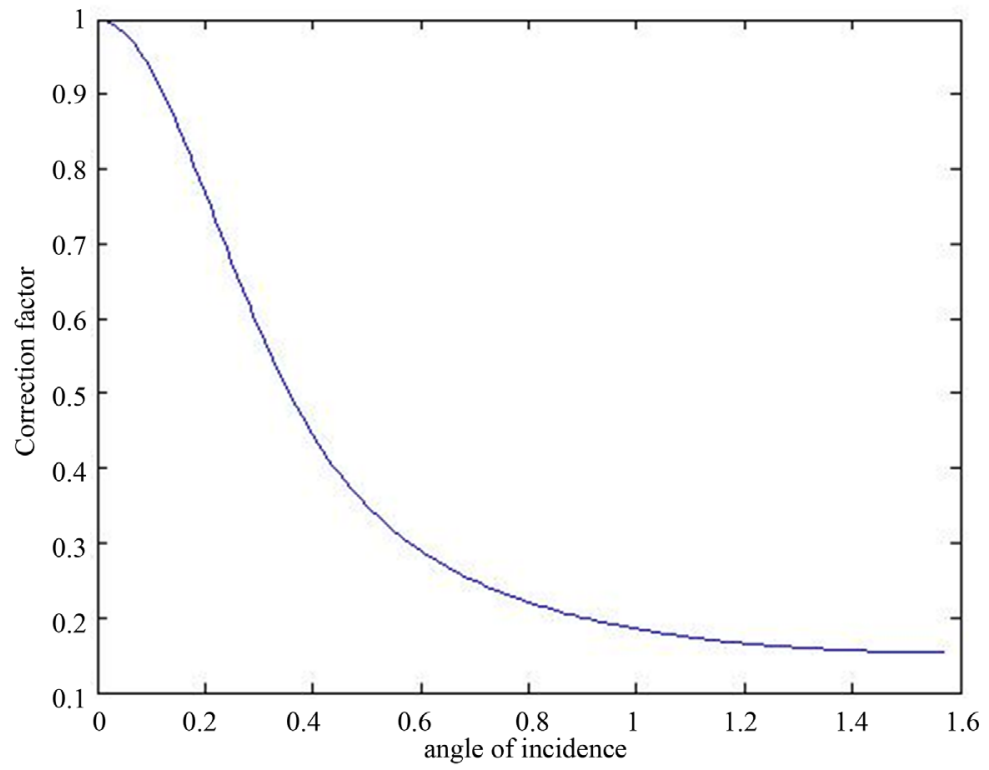

Figure 7. The relationship between angle of incidence and correction factor. 


\section{Model Calculation and Result Analysis}

\subsection{Optimize the Angle and Find the Maximum Number of Hops}

As for S/N ratio explanation [5], the main parameters of the world profile of atmospheric radio noise reported by CCIR-3 22 are the effective noise figure $F_{a}$. It is defined as the ratio of the external radio noise power received by the non-directional short vertical antenna to the noise power generated by the heat source at temperature $T_{0}$ in the unit bandwidth that is the title of the signal to noise ratio [6]:

$$
F_{a}=\frac{P_{n}}{k T_{0} B}
$$

$P_{n}$ is the noise power received by the non-directional short vertical antenna.

$K$ is boltzmann constant; $B$ is the effective noise bandwidth of the receiver.

$W$ is initial signal power.

As for $F_{a}=10 \lg \frac{P_{n}}{W}$, the critical condition is when the noise power is equal to the signal power, so according to the problem (what is the maximum number of hops the signal can take before its strength falls below a usable signal-to-noise ratio (SNR) threshold of $10 \mathrm{~dB}$ ):

$$
W\left(L \times R_{1}\right)^{n}>P_{n}
$$

With $W=100 W$, we can get the remaining power after the reflection with

$$
W_{1}=W\left(L \times R_{1}\right), W_{2}=W\left(L \times R_{1}\right)^{2}, W_{3}=W\left(L \times R_{1}\right)^{3}, \cdots, W_{n}=W\left(L \times R_{1}\right)^{n}
$$

In the case of the incident angle is determined, the maximum number of hops is uniquely determined, we make an interval traversal of the angle and get angle of incidence and the maximum number of hops in Figure 8.

And maximum number is 14 .

The size of the incident angle can affect the radio wave propagation time in the air and thus the reflection intensity, so we can get the relationship between the first reflection intensity and the incident angle in Figure 9.

\subsection{Intensities Comparison of the First Reflections in Calm and Turbulent}

The angle is $57^{\circ}$ from the figure and we can get the first reflection strenghth [8]:

$$
P_{1}=W Q_{1}=7, P_{2}=W Q_{2}=3, P_{1}>P_{2}
$$

\section{Conclusion}

By using original Longley-Rice formula, the minimum loss under the maximum transmission distance and the practicality of radio propagation in the navigation of ships are discussed. The multiple correction parameters is found, and the loss of radio propagation is calculated, which can be used to estimated loss and save 


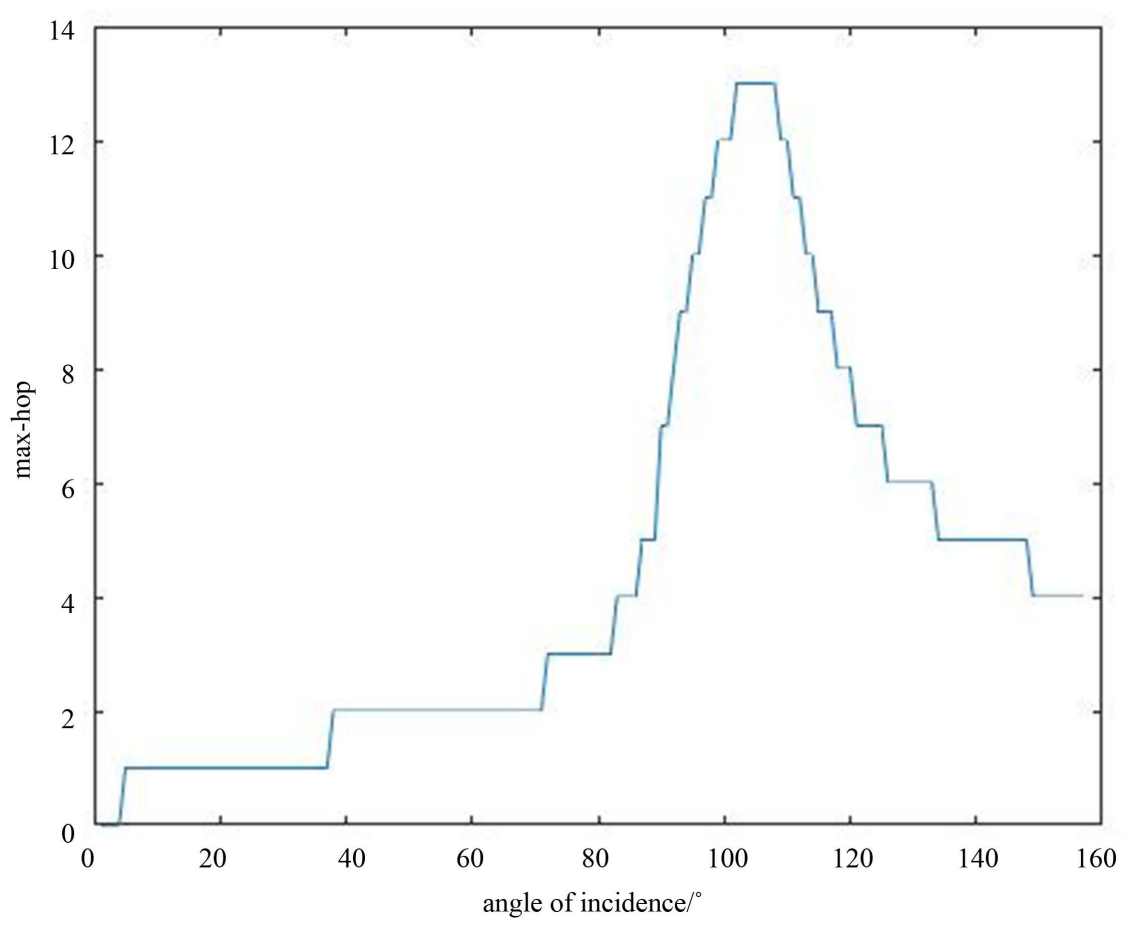

Figure 8. The relationship between angle of incidence and max-hop.

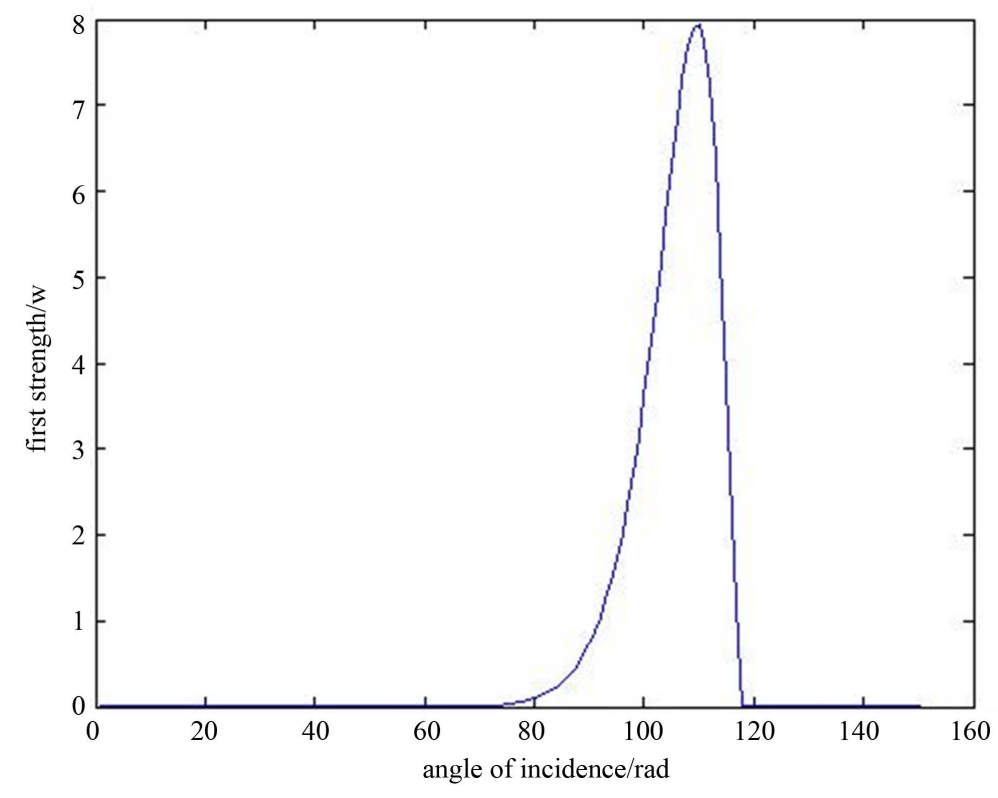

Figure 9. The relationship between angle of incidence and first strength.

energy. The model accurately describes the various types of losses in radio transmission acceptance, with great significance to the communication in the route. In addition to that, our paper is not only used to the situation above, but widely applies to kinds of fields such as marine radar communication, mountain exploration, etc. In more details, the correction factor we propose can also apply to many problems which have a traditional formula without matching situation. 


\section{References}

[1] Li, J.-S. (2014) Study on AIS Sea Wave Propagation Model. Dalian Maritime University, Dalian, 32-45.

[2] Yi, Q.D. (2015) Research on Electromagnetic Wave Propagation model in Sea Area. Hainan University, Hainan.

[3] Fang, H. (2015) Research on Propagation Characteristics and Channel Modeling of Maritime Radio Waves. Hainan University, Hainan, 67-98.

[4] Yu, W.Z., Chi, X. and Ren, J. (2014) Transmission Model of Maritime Mobile Channel Based on ITM. Journal of Electronics, 40, 106-111.

[5] Jin, F. (2015) Radio Tracking Based on the Law of Radio Propagation in the Hilly Areas. Nanjing University of Posts and Telecommunications, Nanjing, 42-51.

[6] Xu C., Qiu, C.-C. and Li, L. (2015) Status and Analysis of Optimum Frequency Selection for Maritime HF Communications. Communications Technology, 1, 17-23.

[7] Zhao, Y.C. (2009) Research and Implementation of Radio Wave Propagation Prediction and Interference Analysis. National University of Defense Technology, Changsha.

[8] Peng, F.F. and Zhou, X.J. (2017) Prediction of Maritime Shortwave Telecommunication Links. Ship Electronic Engineering, 31, 125-127.

[9] Li, Z. (2018) Analysis of Maritime Radio Communication Time Based on Longley-Rice Model. Science and Technology Innovation and Application, 13, 16-17.

[10] Liu, J. (2018) On the Development Trend of Anti-Interference Technology for Ultra-Shortwave Radio Communication. Shandong Industrial Technology, 2, 117-118.

[11] Zhang, X.G. (2018) "Internet + Radio" to Achieve Efficient Radio Supervision. Science and Technology Economics Guide, 26, 192. 